\title{
(Optochemical) control of synthetic microbial co-culture interactions on microcolony level
}

Alina Burmeister ${ }^{1,2}$, Qiratt Akhtar ${ }^{1}$, Lina Hollmann ${ }^{1}$, Niklas Tenhaef ${ }^{1}$, Fabienne Hilgers ${ }^{5}$, Fabian Hogenkamp ${ }^{6}$, Sascha Sokolowsky ${ }^{1}$, Jan Marienhagen ${ }^{1,3}$, Stephan Noack ${ }^{1}$, Dietrich Kohlheyer ${ }^{1,4}$ and Alexander Grünberger ${ }^{2^{*}}$

\footnotetext{
1 Institute of Bio- and Geosciences, IBG-1: Biotechnology, Forschungszentrum Jülich, 52425 Jülich, Germany ${ }^{2}$ Multiscale Bioengineering, Bielefeld University, 33615 Bielefeld, Germany

${ }^{3}$ Institute of Biotechnology, RWTH Aachen University, 52074 Aachen, Germany

${ }^{4}$ Aachener Verfahrenstechnik (AVT-MSB), RWTH Aachen University, 52074 Aachen, Germany

5 Institute of Molecular Enzyme Technology, Heinrich-Heine-University Düsseldorf, Forschungszentrum Jülich, 52428 Jülich, Germany

6 Institute of Bioorganic Chemistry, Heinrich Heine University Düsseldorf, Forschungszentrum Jülich, 52428 Jülich, Germany
} 


\section{Supplemental Figures}

\section{Figure S1}

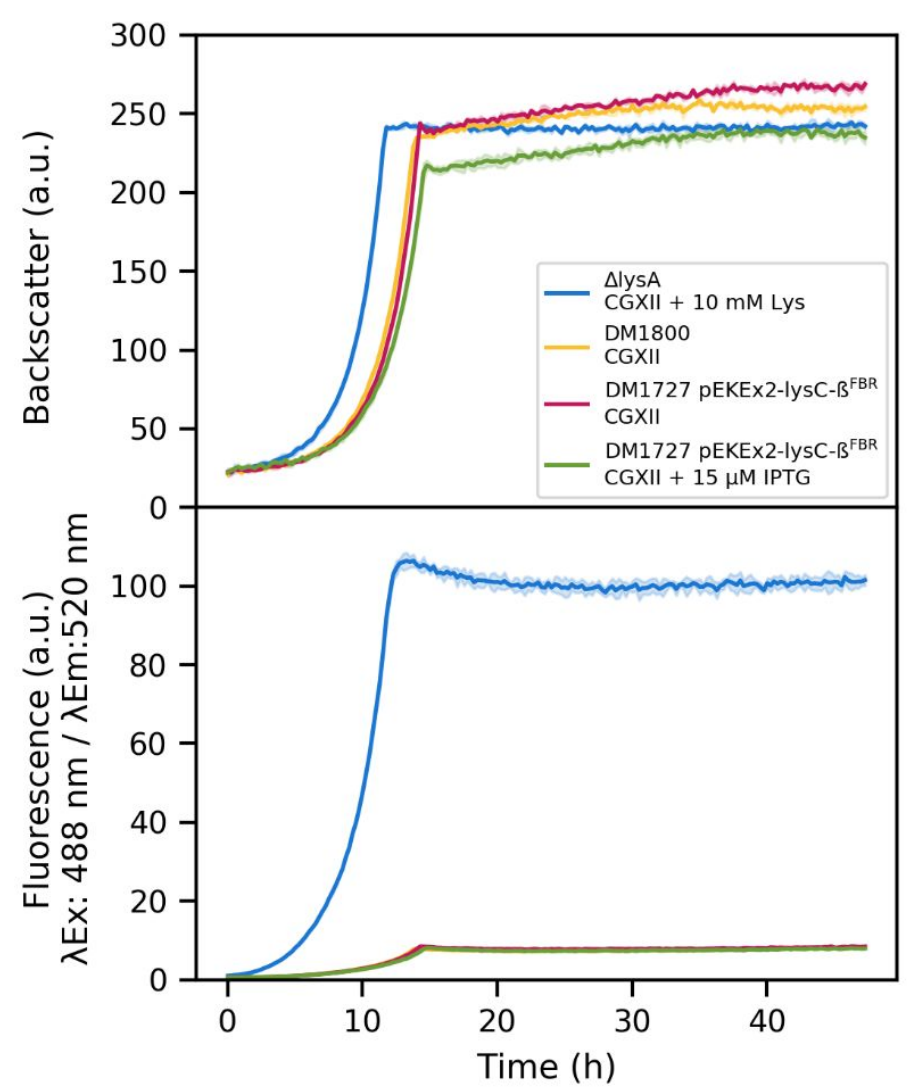

Figure S1 Bulk analysis of co-culture strains using microtiter plate cultivations. Time course of online backscatter and fluorescence data is shown for mono-cultures of strains $\Delta / y s A$ with addition of $10 \mathrm{mM}$ L-lysine, DM1800 and DM1727 pEKEx2-lysC- $\beta^{F B R}$. Mean values (lines) and standard deviations (underlaying areas) were estimated from three independent replicate cultures, respectively. 


\section{Figure S2}

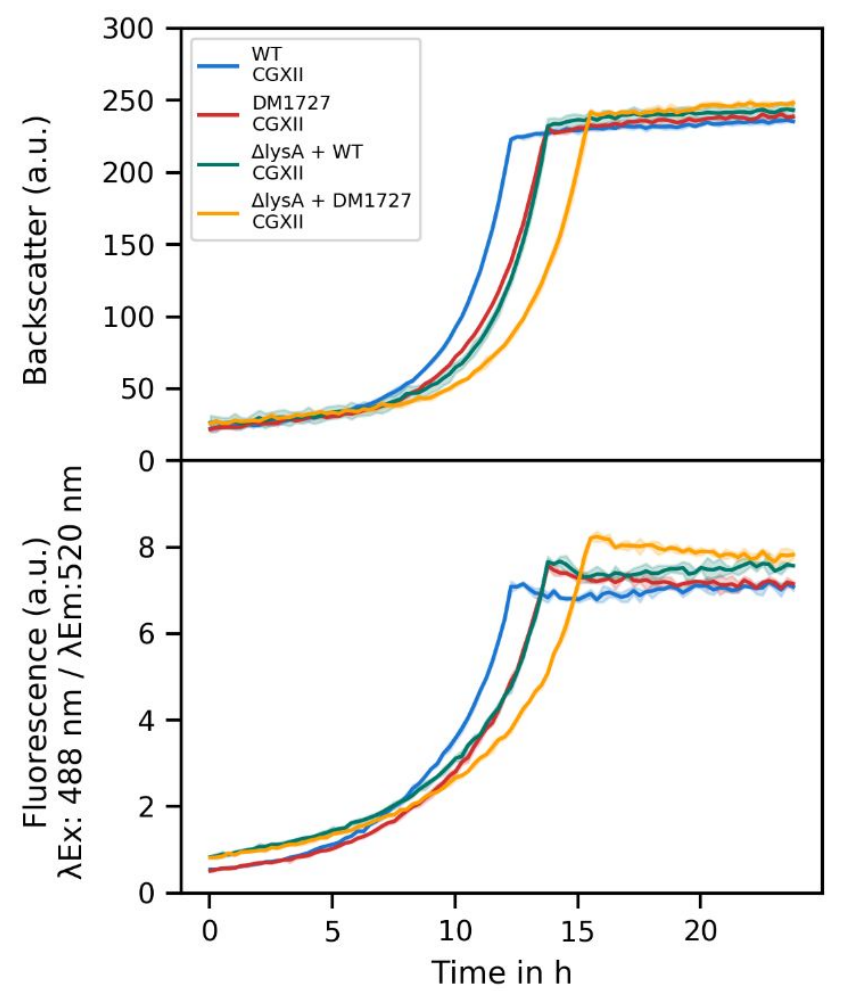

Figure S2 Bulk analysis of co-cultures using microtiter plate cultivations. Time course of online backscatter and fluorescence data is shown for strains $\triangle l y s A$, wild type (WT), and DM1727 in co-culture and separated. Mean values (lines) and standard deviations (underlaying areas) were estimated from three independent replicate cultures, respectively. 


\section{Figure S3}
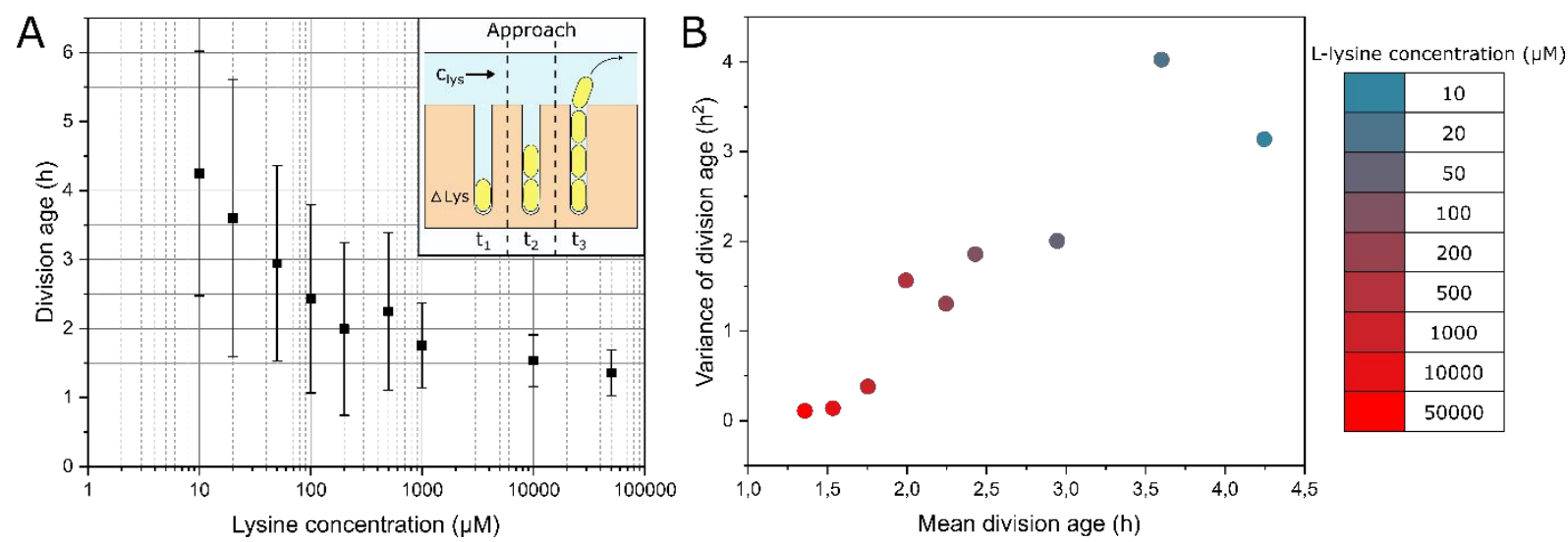

Figure S3 Growth characterization of C. glutamicum $\Delta l y s A$ in mother machines. (A) Cells were cultivated in growth channels with single-cell resolution under a continuous flow of CGXII medium with different L-lysine concentrations. A minimum of $10 \mu \mathrm{M}$ was necessary to enable cell division, but cells show a highly heterogeneous growth behavior under low concentrations. (B) Variance over mean division age for different $L$ lysine concentration. The color of the data points represents the respective L-lysine concentration.

\section{Figure S4}

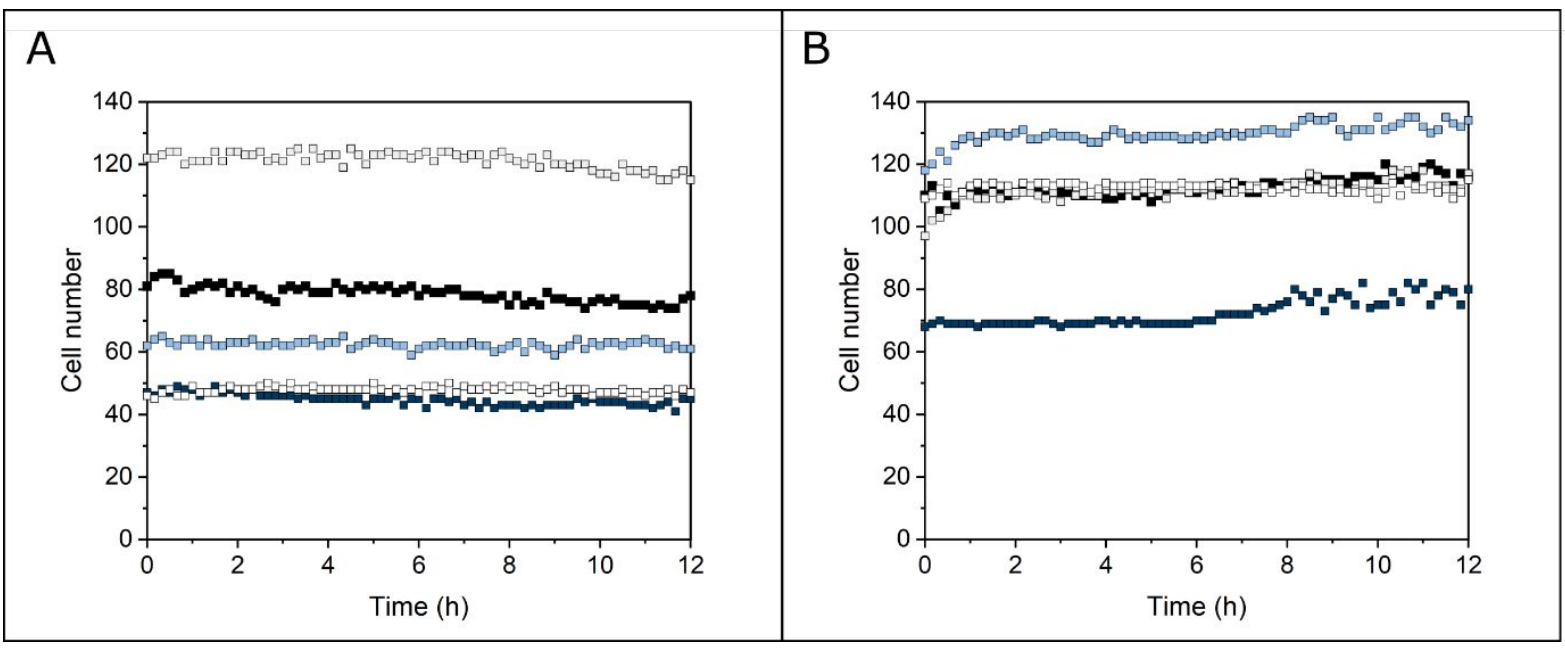

Figure S4 (A) Cell number of C. glutamicum $\triangle$ lysA in co-cultivation with C. glutamicum wildtype in five individual microfluidic growth chambers. (B) Cell number of C. glutamicum $\triangle l y s A$ in co-cultivation with C. glutamicum DM1727 (single pyc mutation) in five individual microfluidic growth chambers. 


\section{Figure S5}

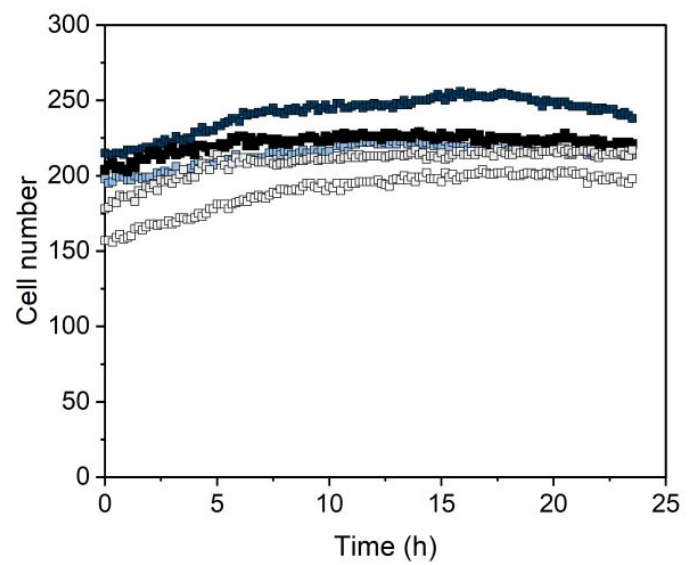

Figure S5 Control experiment of C. glutamicum $\triangle$ lysA in co-cultivation with C. glutamicum DM1727 pEKEx2-lysC$\beta^{F B R}$ without the addition of IPTG in five individual microfluidic growth chambers.

\section{Figure S6}
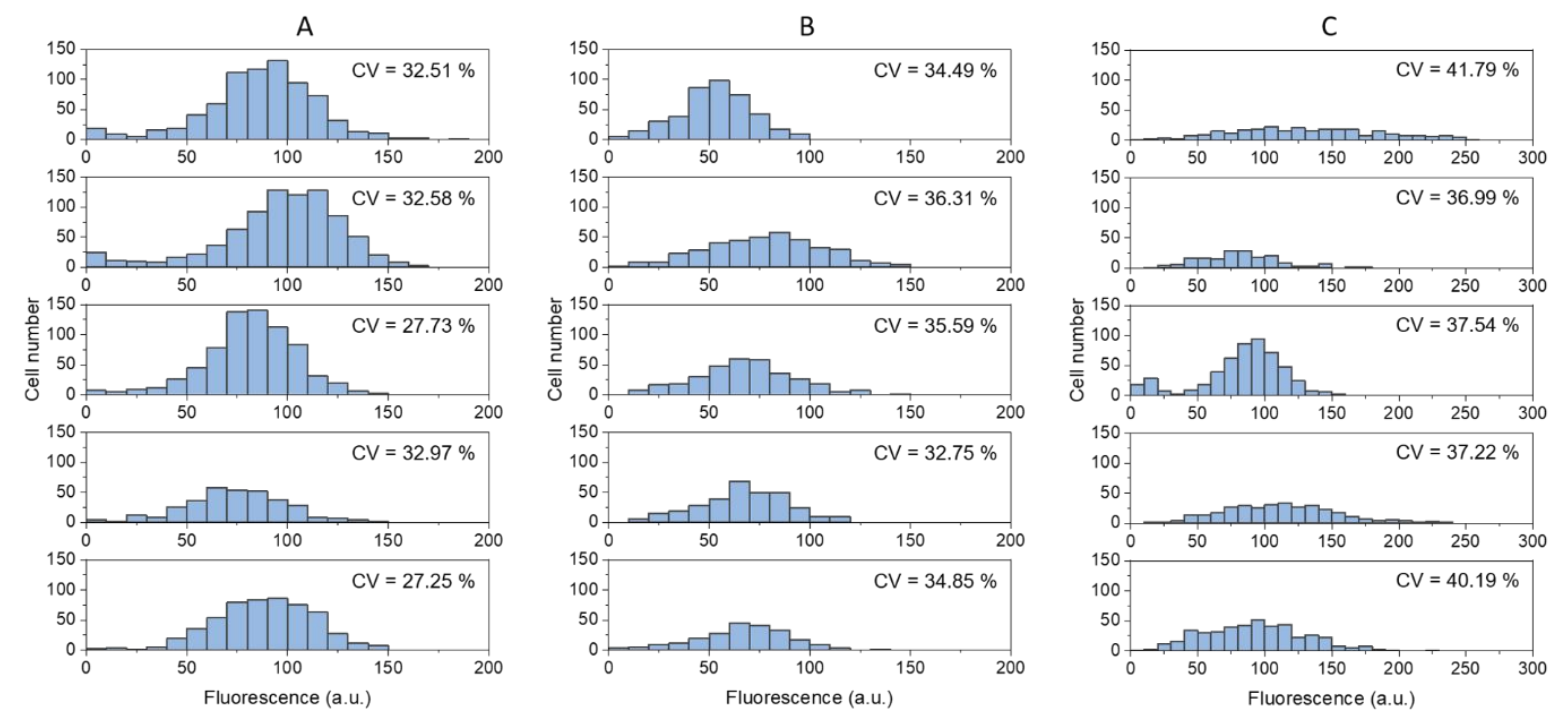

Figure S6 Fluorescence distribution of C. glutamicum $\triangle l y s A$ in co-cultivation. (A) Fluorescence distribution of C. glutamicum $\triangle l y s A$ in co-cultivation with C. glutamicum DM1800. (B) Fluorescence distribution of C. glutamicum $\triangle$ lysA in co-cultivation with C. glutamicum DM1727 pEKEx2-lysC- $\beta^{F B R}$ induced with $15 \mu M$ IPTG. (C) Fluorescence distribution of C. glutamicum $\triangle l y s A$ in co-cultivation with C. glutamicum DM1727 pEKEx2-lysC- $\beta^{F B R}$ induced with $100 \mu \mathrm{M}$ cIPTG and 30 s UV-A exposure. The histograms show fluorescence distributions in five microfluidic co-cultivation chambers after 10 hours cultivation time. For each chamber, the coefficient of variation (CV) was calculated to determine heterogeneity. 


\section{Figure $\mathbf{S 7}$}

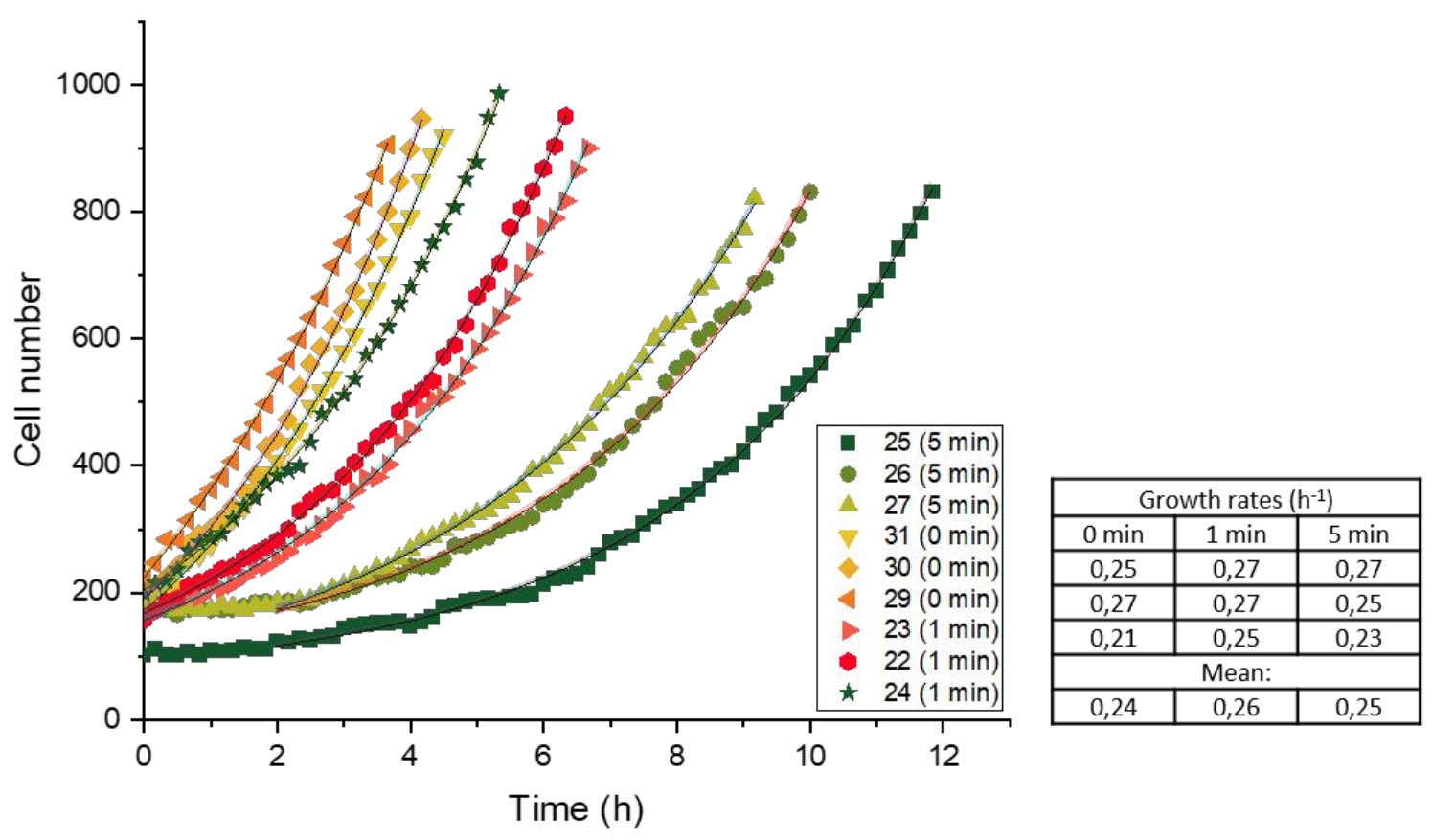

Figure S7 UV-A toxicity test. C. glutamicum cells were exposed to UV-A light inside microfluidic growth chambers for different exposure times. Growth rates were determined to examine a potential negative effect on cell viability. No significant difference in growth rate could be found $(p>0.05)$. 